\title{
Post-Vaccination Immunity Against Measles in Under Twenty-Five-Year-Old Population of Ahvaz, Southwest of Iran
}

\author{
Abdolhussein Shakurnia ${ }^{1}$, Seyed Mohammad Alavi ${ }^{2}$, Reza Norouzirad ${ }^{3, *}$, Amir Arsalan \\ Sarajian ${ }^{4}$, Ghodratollah Shakerinejad ${ }^{4}$ \\ ${ }_{1}^{1}$ Depatment of Immunology, Health Research Institute, Infectious and Tropical Disease Research Center, Ahvaz Jundishapur University of Medical Sciences, Ahvaz, IR Iran \\ ${ }_{2}^{2}$ Department of Infectious disease, Health Research Institute, Infectious and Tropical Disease Research Center, Ahvaz Jundishapur University of Medical Sciences, Ahvaz, IR Iran \\ ${ }^{3}$ Department of Biochemistry, Dezful University of Medical Sciences, Dezful, IR Iran \\ ${ }^{4}$ Department of Health Research Center, Khuzetan Jahade Daneshgahi, Ahvaz, IR Iran \\ ${ }^{*}$ Corresponding author: Reza Norouzirad, Department of Biochemistry, Dezful University of Medical Sciences, Dezful, IR Iran. Tel: +98-9163410049, Fax:+98-6416269534, E-mail: no- \\ rouzirad@gmail.com.
}

Received: August 11, 2012; Revised: December 23, 2012; Accepted: January 16, 2013

\begin{abstract}
Background: Measles is a severe contagious disease, preventable by vaccination. The Ministry of Health and Medical Sciences of Islamic Republic of Iran, has launched a mass Measles vaccination campaign throughout the country from 2003. More than 32 million inhabitants between 5 and 25 years old received the measles vaccine.

Objectives:The purpose of this study was to determine the prevalence of positive measles antibody titers in target groups (subjects under 25 year olds) who had received mass vaccination.

Patients and Methods: We conducted this cross-sectional study on 900 subjects ( 363 males and 537 females), referred to health care and medical center of Ahvaz during 2011. Measles IgG antibody of serum was measured using the ELISA method.

Results: Overall, 821 (91.2\%) of the studied population under 25 years, had immunity to measles. Among girls and boys, $92.2 \%$ and $89.8 \%$ were seropositive, respectively. These differences were not statistically significant between the two sexes $(\mathrm{P}=0.133)$. Anti-measles antibody titer increased with the increase of age from $\leq 5$ to 21 - 25 years (87.3\% in $\leq 5$-year-old group vs. 96.5 in 21 to 25 -year-old group). These differences were statistically significant between the age groups $(\mathrm{P}=0.021)$.

Conclusions: Results indicated that the present vaccination program is insufficient for immunity against measles in this area. The general population immunity was lower than the necessary level for the elimination of measles. Therefore, sero-epidemiological studies are necessary to organize national programs for control and elimination of measles.
\end{abstract}

Keywords: Measles; Immunity; Vaccination; Seroprevalence

\section{Background}

Measles is an acute and highly contagious viral disease which could be prevented by general vaccination. Although an effective and suitable vaccine has been available for the last 30 years, there are annually thousands of deaths due to measles, especially in the developing countries (1-3). Mass catch-up measles vaccination campaigns are recommended by the World Health Organization (WHO) as one of the main strategies to control or eliminate measles. Eastern Mediterranean countries have performed measles overpowering activities during 1997-2010. Following the successful vaccination program for poliomyelitis in 2000 , a program for measles elimination in Iran is in progress according to the recommendation of the world health organization (4).

In Iran, routine immunization of children with one dose (for nine-month infants) of the single measles vac- cine started in 1970 (5). After increasing the immunization coverage in the community which resulted in lower measles virus circulation in the environment and decreasing antibody levels in older people in the community , the epidemiological image of this disease has been changed and thus the measles is being reported more in adults (6).

Stopping the measles transmission in the community needs more than $95 \%$ of immunity coverage (7). Several studies suggest that high immunity level in the community is not possible by the routine vaccination programs (8). Therefore, in such areas which perform routine vaccination in 9 and 15 months old children, the supplement dose should be applied by mass vaccination campaign besides a catch up approach for the susceptible age groups of 5 - 25 years old in Iran, without a history of vaccination. The aim of the expanded program on immunization 
was to achieve a full coverage against more common preventable diseases including measles throughout the country. Several measles outbreaks in Iran showed that Expanded Programme on Immunization (EPI) had some pitfalls. Therefore, Iranian health governor decided to increase the herd immunity via a mass campaign against measles and rubella. Evaluation of immunization coverage (for controlled estimation of susceptible individuals) as well as a surveillance program (for burden control of the disease and virus transmission) are vital for prevention and control of the measles.

Measles eradication has been started from 1994 by catch up vaccination programs in Oman, Kuwait and other eastern Mediterranean countries including Iran (4). It is expected that after the mass campaign, the immunity level in the community reaches to $95 \%$, as in $\mathrm{Ku}$ wait and Tunisia the immunity coverage of measles has been reported as high as 93\%. In Saudi Arabia, after a mass campaign vaccination program in 2000 , the immunity level has been reported 97\% (9, 10). Iran has achieved high population-based immunity to measles through high first- and second-dose routine measles vaccine coverage over the past decade in addition to a nationwide catch-up campaign in 2003 that targeted over 33 million people aging 5 - 25 years which achieved higher than 95\% immunity coverage $(11,12)$. A sero-epidemiological survey, conducted in 2007 , showed that $97.4 \%$ of the population aging 5 - 40 years were immune to measles (13).

\section{Objectives}

The measles mass vaccination campaign in the Islamic Republic of Iran was conducted in the 5 - 25 age groups during December 2003. This study was undertaken to investigate the effectiveness of the campaign in Ahvaz city, by examining the measles IgG seroprevalence after the mass measles vaccination campaign. It is expected that the obtained data help measles elimination in the country.

\section{Patients and Methods}

This cross-sectional survey was performed on 900 healthy subjects younger than 25 years in 2011 in Ahvaz city of Iran. The sample size was determined considering an expected prevalence of measles antibodies to be $90 \%$ in the target groups. Based on this prevalence in the study groups, with $\alpha=0.05$ and desired precision equal to 0.025 , statistical analysis indicated that 900 sera were required. The Sera were collected from healthy subjects referred to health care and medical center in Ahvaz city.

At the time of specimen collection, information regarding date of birth, sex, health status and dates of vaccinations were recorded. Blood samples were obtained by venipuncture of the subjects, who agreed to take part in this study and signed the consent form. Subjects with a previous history of contagious disease and persons having any immunocompromising conditions were excluded. The study was approved by ethics committee of Ahvaz Jundishapur University of Medical Sciences. Measles Immunoglobulin G (IgG) antibodies were determined using an enzyme-linked immunosorbent assay (ELISA) licensed commercial kit (Vircell, Spain). ELISA results were expressed as optical density (OD), and then the OD values were categorized as positive or negative using fixed cutoffs. An optical density ratio $\geq 11.0$ was interpreted as positive while value $<11.0$ was interpreted as negative for IgG measles.

The specificity of the kit was $94 \%$ while its sensitivity was 97\%. Data were analyzed using the Statistical Package for Social Sciences software (SPSS - 18). P value less than 0.05 was considered as significant. The percentages of positive and negative sera were calculated. Seropositivity prevalence among different groups was compared using the chi-square test.

\section{Results}

A total of 900 subjects including 363 (40.3\%) males and 637 (59.7\%) females were studied. All were in good health, ranging from 1 to 25 years old (mean of $12.7 \pm 6.5$ ). AntiMeasles antibody titer was positive in 821 (91.2\%) and negative in 79 (8.8\%). From 363 males, 326 cases (89.8\%) and from 537 females, 495 cases (92.2\%) were seropositive. Using chi-square test, no significant statistical difference was detected between the immunity level of males and females $(P=0.133)$. Similarly, there were no statistical differences among the studied age groups regarding sex. The prevalence of Anti-measles antibodies was higher in both genders (93.1\% vs. 97.6\%) in the 21 - 25-year-old group, while in the other age group, the prevalence of seropositivity was lower in both genders. Table 1 shows the prevalence of seropositivity and the mean antibody titer in males and females of different age groups.

Regardless of gender, the prevalence of seropositivity in $\leq 5,6-10,11-15,16-20$ and $21-25$ age groups was: $87.3 \%, 87.7 \%, 90.9 \%, 93.7 \%$ and $96.5 \%$, respectively. There was statistically significant differences in seropositivity proportions regarding the age groups $(\mathrm{P}=0.021)$. The female mean antibody level was slightly higher than that of observed in males, but it was not statistically significant $(29.98 \pm 25.5$ vs. $27.41 \pm 23.5 ; \mathrm{P}=0.120)$ (Table 1$)$. The age group mean antibody level was higher than that of observed in $16-20 ; 31.6 \pm 26.7$ vs. $28.9 \pm 14.7$ in $21-25,28.5 \pm$ 24.1 in $11-15,29.6$ in $6-10$ and $26.5 \pm 23.3$ in $\leq 5$ years. These differences were not statistically significant (0.082).

Figure 1 shows the frequency distribution of various ages with relation to the immune status and mean level of measles antibody. There was a positive correlation between age group and seropositivity rate, meaning that with an increase in age, the rate of seropositive results elevated $(r=0.036, P=0.274)$. 
Shakurnia A et al.

\begin{tabular}{|c|c|c|c|c|}
\hline Gender & Age Group & Seropositive, No. (\%) & OD, Mean (SD) & Total \\
\hline \multicolumn{5}{|l|}{ Male } \\
\hline & $<5$ & $55(87.3)$ & $23.9(21.7)$ & 63 \\
\hline & $6-10$ & $90(88.2)$ & $28.9(25.3)$ & 102 \\
\hline & $11-15$ & $76(90.5)$ & $25.4(19.8)$ & 84 \\
\hline & $16-20$ & $78(91.8)$ & $31.3(21.7)$ & 85 \\
\hline & $21-25$ & $27(93.1$ & $23.7(14.2)$ & 29 \\
\hline & Total & $326(89.8)$ & $27.4(23.6)$ & 363 \\
\hline \multicolumn{5}{|l|}{ Female } \\
\hline & $<5$ & $41(87.2)$ & $29.8(25.1)$ & 47 \\
\hline & $6-10$ & $103(87.3)$ & $30.2(29.1)$ & 118 \\
\hline & $11-15$ & $93(91.2)$ & 31.1() 26.9 & 102 \\
\hline & $16-20$ & $175(94.6)$ & $31.7(26.1)$ & 185 \\
\hline & $21-25$ & $83(97.6)$ & $24.7(15.1)$ & 85 \\
\hline & Total & $495(92.2)$ & $29.9(25.5)$ & 537 \\
\hline
\end{tabular}

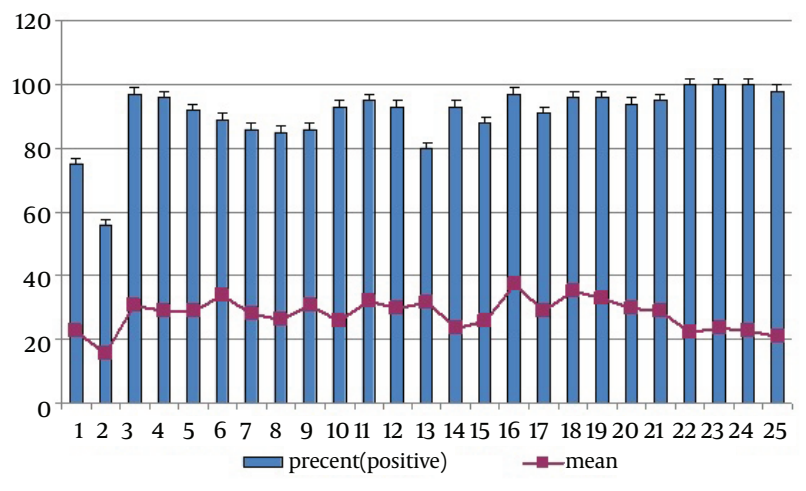

Figure 1. Age-Specific Seropositive Prevalence and Mean Level of Measles Antibody

\section{Discussion}

In this study, we measured anti-measles IgG antibody of under 25 years cases to determine the proportion of sero-positivity. Of the studied population, $91.8 \%$ were sero-positive and were immunized against measles, while more than $8 \%$ were not. According to the eastern Mediterranean countries plan of action for measles elimination (WHO, January 2003), immunity of 95\% in the population is necessary to interrupt transmission and eliminate measles (14). The results of this study reveal that the overall measles seroprevalence rate (91.8\%) of this area should be further increased to achieve the abovementioned goal. This level of immunity in the population could be alarming and is potentially susceptible to incidence of measles epidemiology in the population.

Immunity level of less than 90\% in the children below five, and also six to 10-year-old groups, represents the disaster. In addition, identified groups with less than 95\% immunity might be at a high risk of leading to future outbreaks. These finding are helpful for public health authorities in order to be aware of the immunity level and regularly perform measles surveillance. As we don't know about the measles vaccination in the target groups, low immunity in this population could be due to low vaccination level.

Recent successes of measles transmission interruption in the World Health Organization region of the Americas, most European countries and selected countries in other regions, provide evidence for the feasibility of global eradication (15). In developing countries, reaching such high level of vaccination coverage was not possible with the routine programs and thus needed to be supplied with a general vaccination, such as a catch upa catch-up campaign, providing measles vaccine to all children regardless of prior history of immunization or disease, in the susceptible age groups.

Nowadays, sero-pidemiological studies before and after general vaccination programs in most countries is showing an immunity level of more than 95\% (16-18). Several studies have been performed to evaluate the presence of anti-measles antibody after the mass campaign vaccination in different regions of Iran. Two studies in Shiraz and Tehran after one and five years of vaccination have revealed the presence of antibody in $87.5 \%$ and $88.5 \%$ of the studied populations $(19,20)$. This value was $90.3 \%$ in the survey of Savadkoohi et al. in Babol (21), 91.6\% in Salimi et al. evaluation in Tabriz (18) and 97.4\% in Esteghamati et al. study in Tehran (17). The results of these studies were in accordance with our observations in the Ahvaz city. Seroepidemiological studies have demonstrated that the universality of vaccination coverage has an important role in the development of antibody titer for measles, especially in high-age groups $(22,23)$. The reason for such high level of immunity in the higher age groups is the efficacy of mass campaign for vaccination of measles-rubella, and successful expanded program on immunization. 
The highest seropositive cases were identified in the 21 25 age groups (96.5\%). Such an increase in the prevalence of high-level seropositivity was observed in a study in Urmia and Tabriz, perhaps reflecting more effective control of measles virus in Iranian populations $(18,23)$. A serological survey in Hong Kong showed that people aging 20 years and higher had a high level of immunity against measles (16).

The same finding was also observed in Turkey where the immunity for measles was lower among preschool children than older age groups (24). Several prospective serological studies demonstrated a gradual progressive decline in measles antibody titer during several years after vaccination. On the contrary, some other surveys showed that there was a significant increase in the antibody titer in older age groups $(16,24,25)$. This means that during the mass campaign vaccination program in a community measles immunity increases by age. According to the literature, before the mass campaign, immunity level in the high aged groups was lower, so the incidence rate was higher $(26,27)$.

Our finding suggesting that after a suitable mass campaign vaccination for measles control, the epidemiological outline for this disease has changed, as the immunity level has increased in the high aged groups.

Generally, the results revealed that seropositive prevalence against measles was relatively low in this area, so there is a possibility that measles antibody levels were insufficient to protect against measles, especially in the lower age groups. However, the immunity level for measles increased with age and reached to the maximum in the age group of $21-25$. This finding suggests that mass campaign vaccination of measles is successful for its elimination. According to the immunity coverage under $95 \%$ in the studied population after eight years of mass campaign vaccination for measles, it is recommended that measles sero-epidemiologial study should be repeated every four to five years.

\section{Acknowledgements}

None declared.

\section{Authors' Contribution}

None declared.

\section{Financial Disclosure}

None declared.

\section{Funding/Support}

This study was supported by Ahvaz Jundishapur University of Medical Sciences and Khuzestan Jahad Daneshgahi.

\section{References}

1. Christie AS, Gay A. The Measles Initiative: moving toward measles eradication. J Infect Dis. 2011;204 Suppl 1:S14-7.

2. Hall R, Jolley D. International measles incidence and immunization coverage. J Infect Dis. 2011;204 Suppl 1:S158-63.

3. Kaninda AV, Legros D, Jataou IM, Malfait P, Maisonneuve M, et al. . Meales vaccine effectiveness in standard early immunization strategies, Niger, 1995. Pediatr Infect Dis J. 1998;17:1034-39.

4. Gaafar T, Moshni E, Lievano F. The challenge of achieving measles elimination in the Eastern Mediterranean Region by 2010.J Infect Dis. 2003;187(Supplement 1):S164-71.

5. Mokhtari Azad T, Mahmoodi M, Hamkar R, et al. . [Epidemiologic feature of measles in Iran, 1995-96]. Hakim Res J. 2000;1:19-26.

6. Griffine DE. Measles Virus. In: Kinpe DM, Howley PM editors Fields Virology. 4 ed. United State America: Lippincott Williams \& Wilkins; 2001. p. 1401-41.

7. Muller CP. Measles elimination: old and new challenges? Vaccine. 2001;19(17-19):2258-61.

8. Bataweel NA, Al-Robasi AA, Hasoon S, Al-Jabri AA, Sallam TA. Effect of first and second doses of Measles vaccine on immunity to measles among infants in sana'a city, Yaman.J Arab Board Health Special. 2012;13(1):10-15.

9. Naouri B, Ahmed H, Bekhit R, Teleb N, Mohsni E, Alexander JP, Jr Progress toward measles elimination in the Eastern Mediterranean Region. J Infect Dis. 2011;204 Suppl 1:S289-98.

10. Khalil MK, Al-Mazrou YY, AlHowasi MN, Al-Jeffri M. Measles in Saudi Arabia: from control to elimination. Ann Saudi Med. 2005;25(4):324-8.

11. Zahraei SeyedMohsen, Gouya MohamadM, Azad TalatMokhtari Soltanshahi Rambod, Sabouri Azam, Naouri Boubker, et al. Successful control and impending elimination of measles in the Islamic Republic of Iran. J Infect Dis. 2011;204(suppl 1):S305-11.

12. Zahraei SM, Sadrizadeh B, Gouya MM. Eradication of poliomyelitis in Iran, a historical perspective. Iran J Public Health. 2009;38(Suppl.1).

13. Majdzadeh R, Moradi A, Zeraati H, Sepanlou SG, Zamani G, Zonobi V. Evaluation of the measles-rubella mass vaccination campaign in the population covered by Tehran University of Medical Sciences. East Mediterr Health J. 2008;14(4):810-7.

14. Morgan OliverWC. Following in the footsteps of smallpox: can we achieve the global eradication of measles? BMC Int Health Hum Rights. 2004;4(1):1-5.

15. Peltola Heikki, Jokinen Sari, Paunio Mikko, Hovi Tapani, Davidkin Irja. Measles, mumps, and rubella in Finland: 25 years of a nationwide elimination programme. The Lancet Infect Dis. 2008;8(12):796-803.

16. Chuang SK, Lau YL, Lim WL, Chow CB, Tsang T, Tse LY. Mass measles immunization campaign: experience in the Hong Kong Special Administrative Region of China. Bull World Health Organ. 2002;80(7):585-91.

17. Esteghamati A, Gouya MM, Zahraei SM, Dadras MN, Rashidi A, Mahoney F. Progress in measles and rubella elimination in Iran. Pediatr Infect Dis J. 2007;26(12):1137-41.

18. Salimi V, Mokhtri-azad T, Gooya MM, Hamkar R, Esteghamati AR, Varshochiyani SA, et al. Seroepidemiology of measles in 5-25-year-old age group before measles/rubella mass vaccination campaign in Tabriz (2003). Med J Tabriz Univ. 2006;28(2):77-82.

19. Jonaidi Jafari N, Ranjbar R, Kakaei M, Ghorbani GhA, Izadi M, Khosravi A, et al. [The Evaluation of Anti Measles IgG Antibody Level Five Years After Mass Vaccination of Measles- Rubella Among The Medical Students of Baqiyatallah University of Medical Sciences]. Sci J Ilam U Med Sci. 2010;18(1):34-9.

20. Pourabbas B, Ziyaeyan M, Alborzi A, Mardaneh J. Efficacy of measles and rubella vaccination one year after the nationwide campaign in Shiraz, Iran. Int J Infect Dis. 2008;12(1):43-6.

21. Savad Koohi R, Hajian K, Noori NA, Attarieh M. [Measurement of Measles antibody in 157 children aged 4-6 years referred to Amirkola Children Hospital, 2003-2004]. J Babol Univ Med Sci. 2006;8(3):55-9.

22. Shamsizadeh A, Nikfar R, Makvandi M, Hakimzadeh M, Alisamir M, Ziaei T. Seroprevalence of measles, mumps and rubella Antibodies in 18 months and 6.5 years old children: 6 months after measles-mumps-rubella (MMR) vaccination. Jundishapur J Microbiol. 2012;5(4):578-81. 


\section{Shakurnia A et al.}

23. Yekta Z, Porali R, Taravati MR, Salary Sh, Khalily F, Shahabi S. Measles IgG sero-prevalence and its attributable factors in 5-25-yearold cases prior mass vaccination campaign in Urmia, northeastern Iran. Iran Red Crescent Med J. 2007;9(1):28-35.

24. Gozalan A, Korukluoglu G, Kurtoglu D, Miyamura K, Yilmaz N, Morita M, et al. Measles seroepidemiology in 3 cities in Turkey. Saudi Med J. 2005;26(12):1971-7.

25. Glass K, Grenfell BT. Waning immunity and subclinical measles infections in England. Vaccine. 2004;22(29-30):4110-6.

26. Gidding HF, Gilbert GL. Measles immunity in young Australian adults. Commun Dis Intell Q Rep. 2001;25(3):133-6.

27. Emami Naeini A, Ghazavi N, Emami Naeini S. Measurement of serum antibody Titer against Measles in a group of adults receiving Measles-Rubella mass vaccination.J Isfahan Med Sch. 2011;28(114):854-8. 